\title{
Intelligent Buildings: Foundation for Intelligent Physical Agents
}

\author{
Francisco J. Mora Lizán ${ }^{1}$, Carlos Rizo Maestre ${ }^{2}$, \\ ${ }^{1}$ (Departamento de Ciencia de la Computación e IA. Universidad de Alicante. Spain) \\ ${ }^{2}$ (Instituto del Agua y Ciencias Ambientales. Universidad de Alicante. Spain)
}

\section{ABSTRACT}

FIPA is an IEEE Computer Society standards organization that promotes agent-based technology and the interoperability of its standards with other technologies. In the design phase of Intelligent Buildings, it is essential to manage many services and facilities, to do this, multi-agent systems are a good tool to manage them. In this paper, we will gereneral description of the features and elements of multiagent systems described by Foundation for Intelligent Physical Agents (FIPA). Secondly, we will focus on the architectures of these multiagent systems. And finally, we will propose a multi-agent system design to see the application in the design of a detached house where the lighting, air conditioning and security systems will be integrated.

Keywords - Architecture, Intelligent Building, Multiagent Systems

\section{INTRODUCTION}

In the field of intelligent building design, we must have mechanisms to integrate sensors, actuators, different automatic control systems and information capture. MultiAgent Systems (MAS) form a community of interdependent elements that act individually. From the moment that a group of elements, in this case agents, assume to act in group forming systems, the capacity to negotiate and to coordinate different tasks is necessary.

In many cases the use of individual agents is not just as suitable for all situations that occur in practice. Solving a problem using an individual agent causes major constraints. An individual agent requires an enormous amount of knowledge to solve complex problems. In the worst case, the problem can be so complex that an agent cannot find a useful solution. Even when the individual agent can solve a problem, it always presents a bottleneck in terms of speed, reliability, flexibility and modularity. Multiagent systems offer a method to avoid the problematic situations described. In a multi-agent system, several independent autonomous agents are active. Each of these agents is dedicated to their own objectives and only contacts the other agents to obtain information, or to contribute to a coordinated solution of a general problem. In both situations, each individual agent has a specific task for which it is adequate and whose solution does not exceed its capabilities. This allows for the processing of complex problems.

In a building, we can find different subsystems (security, air conditioning, lighting, multimedia, control etc), each with specific objectives and in turn all must work together to achieve a common goal.

Multi-agent systems provide a great advantage: they allow the integration of existing agents into a large system. Therefore, solving a problem does not require the design and development of a new specialized agent, instead, the knowledge of existing agents can be used by combining them into a multi-agent system and allowing them to work together to solve the problem.

The multiagent systems (MAS) constitute a field of research that compose a wide set of applications within Intelligent Buildings, their construction [1], sensor and control of buildings [2], [3], maintenance of Intelligent Buildings [4]. A modern approach to Architectural Construction involves considering MAS as elements linked to the architectural design of buildings [5]. In this sense, a multidisciplinary approach to architectural design is essential [6], the Building Information Models [7] supplemented with the MASs constitute powerful systems to holistic design of buildings [8].

\section{FOUNDATION FOR INTELLIGENT PHYSICAL AGENTS. FIPA}

FIPA [9] is an IEEE Computer Society standards organization that promotes agent-based technology and the interoperability of its standards with other technologies. FIPA was originally formed as a Swiss based organization in 1996 to produce software standards specifications for heterogeneous and interacting agents and agent based systems. FIPA, the standards organization for agents and multi-agent systems was officially accepted by the IEEE as its eleventh standards committee on 2005.

From the structural point of view, the FIPA standard defines a series of key elements, shown in Fig. 1.:

1. An Agent Platform (AP) provides the physical infrastructure in which agents can be deployed. The AP consists of the machine(s), operating system, agent support software, FIPA agent 
management components (DF, AMS and MTS) and agents.

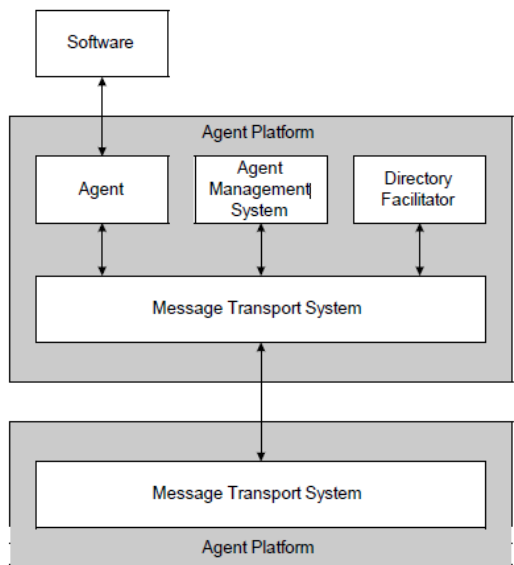

Figure 1. FIPA Management Reference Model.

2. A Directory Facilitator (DF) is an optional component of the AP, but if it is present, it must be implemented as a DF service. The DF provides yellow pages services to other agents. Agents may register their services with the DF or query the DF to find out what services are offered by other agents. Multiple DFs may exist within an AP and may be federated.

3. An Agent Management System (AMS) is a mandatory component of the AP. The AMS exerts supervisory control over access to and use of the AP. Only one AMS will exist in a single AP. The AMS maintains a directory of AIDs which contain transport addresses (amongst other things) for agents registered with the AP. The AMS offers white pages services to other agents. Each agent must register with an AMS to get a valid AID. There are three ways in which an agent can be registered with an AMS: the agent was created on the AP, the agent explicitly registered with the AP and the agent migrated to the AP, for those APs which support agent mobility.

4. A Message Transport Service (MTS) is the default communication method between agents on different APs. FIPA is concerned only with how communication is carried out between agents who are native to the AP and agents outside the AP. Agents are free to exchange messages directly by any means that they can support.

It should be noted that the concept of an AP does not mean that all agents resident on an AP have to be co-located on the same host computer. FIPA envisages a variety of different APs from single processes containing lightweight agent threads, to fully distributed APs built around proprietary or open middleware standards.
FIPA defines the specification of a language for the communication between agents (ACL) in which different types of content are represented: SL (Semantic Language), CCL (Constraint Choice Language), KIF (Knowledge Interchange Format) And RDF ("Resource Description Framework"). In this paper, we are not going to extend more in this part of communications, but we will do it in the structural one.

\section{AGENT ARCHITECTURES}

There are many types of intelligent agents, each of them developing the tasks for which it has been created. And while for some time becomes a critical resource, others will be able to make more rational decisions by having enough time and knowledge to do so. Some agents will be more complex and intelligent than others. Therefore, there is no single ideal architecture for intelligent agents. The concrete structure of the architectures will depend on the tasks and the environment where they are developed. We are going to analyze some architectures that have served as reference and that can be representative of the wide range of possibilities that exist.

Reactive architectures are based on a close relationship between perception and action. They work well in real-time environments since they are computationally economical.

Reactive agents do not have a symbolic model of their environment. The ability to perform complex reasoning processes is also omitted. The reason for these restrictions is in the creation of compact, fault tolerant, and above all, flexible agents.

The Fig. 2 shows the fundamental architecture of the reactive agents that correspond to a simple stimulus / response system. The sensors collect the information, send it to the corresponding competition modules, producing a reaction as output in the same, which is transmitted to the outside by means of actuators.

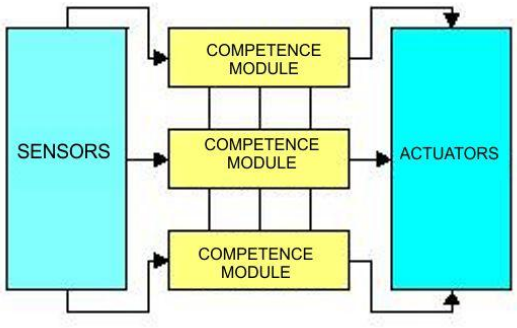

Figure 2: Reactive agent architecture

Deliberative architectures follow the current of symbolic AI, which is based on the hypothesis of the physical-symbol systems enunciated by Newell and Simons, according to which a system of physical symbols capable of 
manipulating symbolic structures can exhibit intelligent behavior. In order to be able to work at Newell's knowledge level, our problem will be how to describe the objectives and means of satisfying them, and how to translate the level of knowledge at the symbolic level.

Decisions are made using deductive mechanisms: Pattern matching and various logical formalisms.

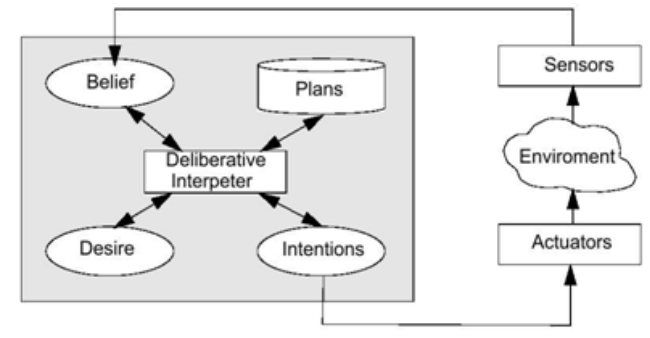

Figure 3: Architecture of deliberative agents

Deliberative agent architectures (Fig. 3) are usually based on the classical artificial intelligence planning theory: given an initial state, a set of operators / plans, and an objective state, the agent's deliberation is to determine which steps to chain To achieve its goal, following a top-down approach.

In BDI ("Belief-Desire-Intention") architectures, decision-making takes place on a process of reasoning based on the agent's beliefs about the world and taking into account intentions and actions.

The basic components of this architecture are the beliefs, desires and intentions of the agent; The functions that represent their deliberation; and the reasoning of ends and means. Practical reasoning involves two important processes: deciding what goals to achieve, a process known as deliberation; and how these goals will be achieved, a procedure called means-ends reasoning. The decision process typically begins by trying to understand what options are available; once this set of alternatives has been generated, one must choose between them and commit with one; this chosen option becomes an intention, which determines the actions of the agent. Intentions locate the agent's future practical reasoning; when one has a particular intention, all those options that are inconsistent with intention are discarded. In addition, once an intention is adopted, the agent must persevere ("persist") in it, it must only rectify it when the reason for which it had the intention has changed; or when the agent knows with certainty that he will not be able to comply with it.

Finally, intentions are closely related to beliefs about the future. When he has an intention, the agent should at least believe that he has a great chance of complying with it.
Hybrid Architecture "Touring Machines" combine deliberative and reactive aspects, by combining reactive modules with deliberative modules. The reactive modules are responsible for processing the stimuli that do not require deliberation, while the deliberative modules determine what actions must be performed to satisfy the local and cooperative objectives of the agents.

The Hybrid Architecture "Touring Machines" are organized by horizontal layers that produce activities. This means that each layer constantly produces suggestions about the actions that the agent should carry out. The reactive layer provides a more or less immediate response to changes in the environment. It is implemented as a set of action-situation rules as shown in Fig. 4.

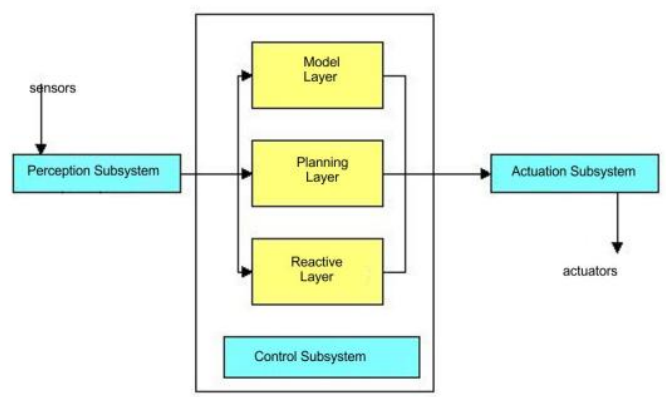

Figure 4: Hybrid Architecture "Turing Machine"

The planning layer serves to provide a proactive agent behavior. Under normal circumstances, the planning layer is responsible for deciding what the agent does. Use a set of skeletons of plans called schemes. These schemes are essentially hierarchically structured plans that the agent elaborates at runtime to decide what to do. To carry out a goal, the planning layer tries to find a scheme that corresponds to that goal. This schema will contain sub-goals, which the planning layer uses to find other schemas that correspond with them.

The modeling layer represents the various entities of the world (including the agent itself, as well as other agents). Predicts conflicts between agents and generates new objectives to resolve these conflicts. These new objectives are passed on to the planning layer that looks for schemes that satisfy them.

The three control layers are embedded in a control subsystem, which decides which of the layers will have control over the agent. This control subsystem is implemented as a set of control rules. These control rules can suppress sensor information for some layer or censor the actions of some layer.

"InteRRaP" is an example of a vertically layered and two-step split agent architecture, as shown in Fig. 5. 


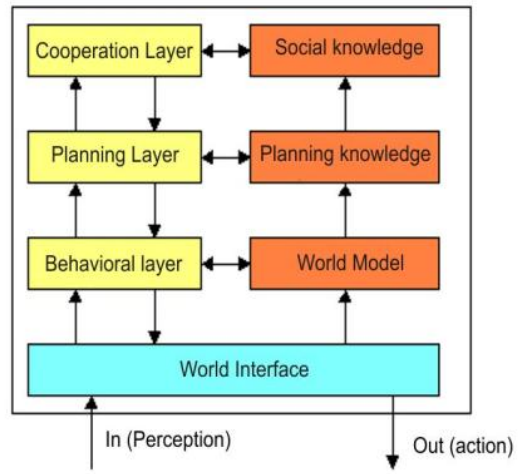

Figure 5: InteRRaP Architecture

As in the "Touring Machines" have three layers of control. In addition, the purpose of each "InteRRaP" layer seems to match the corresponding layer of the "Touring Machine". Each layer is associated with a knowledge base, for example, a representation of the world suitable for each layer. These knowledge bases represent the agent and his environment at different levels of abstraction. The knowledge base at the highest level represents the plans and actions of the other agents in the environment; the knowledge base of the middle level represents the plans and actions of the agent himself; and the bottom-level knowledge base represents information about the environment. The explicit introduction of these knowledge bases distinguishes the "InteRRaP" from the "Touring Machines". The way of the different layers in "InteRRaP" work to produce the behavior also differs from the "Touring Machines". The main difference is the way of the layers interact with the environment. In "Toruing Machines" each layer had as input the perceptions of the environment and as an output an action in the environment. This introduced the need for a control module to deal with conflicts. In "InteRRaP", the layers interact with the rest to achieve the same purpose.

\section{APPLICATION OFARCHITECTURES.}

In our case, we will propose a multi-agent system for a single-person home, where we will use 3 reactive agents for the safety, air conditioning and lighting subsystems, and one with the Touring Machines architecture for global planning and control as shown in Fig. 6. The system will be completed by an agent that acts as an external interface with the users (dashboard), a service agent (ADF) and an agent control agent (AMS).

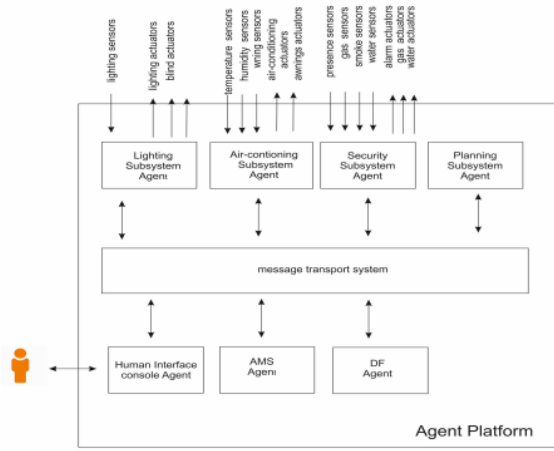

Figure 6: Multiagent System in Intelligent Building

The reactive agent of the safety subsystem receives information directly from the presence, gas, smoke and water sensors. In the case of detection of leaks of water or gas will cut the servo valves of such systems. In the event of a fire, it could start the alarm and the fire system of the house.

For the air conditioning system, we will use another reactive agent where its sensors will collect the data of temperature, humidity of the different rooms and external wind and interectuará with the actuator modules of the air conditioning and awnings.

The latest reactive agent in our system will be responsible for lighting. This agent will obtain information of the interior and exterior light, and indirectly of the presence sensors that the agent of the security system has obtained. Interact with the actuators of blinds and interior lighting. We could also make requests to the air conditioning agent to act on the awnings, requesting for example to raise the awning to get more ambient light.

A very important agent in our system will be with a planning and control agent that uses the Touring Machine architecture. The mission of this agent will be to maintain the security of the house, to satisfy the needs of the inhabitants of the house (comfort) and to promote energy saving. It will be very important to define the desired plans and activities. This agent will send plans and rules to the different agents of the platform (subsystems).

We could have several agents forming each subsystem, the ADF agent would be the one that would inform the rest of the services that each of these agents possesses. In our example the lighting agent would ask the ADF if there is an agent in charge of the service of awnings, the ADF would answer that there is one, and the air conditioning agent will make the necessary requests if necessary.

Remember that all communication between agents will do through the "message transport system" using the agent communication language defined by FIPA. 


\section{CONCLUSION}

In this paper, we have developed the different agent architectures, applying these concepts in the design of a multi-agent system for a house where several subsystems (lighting, air conditioning, safety and control) are integrated. For each of them an architecture has been selected, attesting to its characteristics and needs. This system has been provided with a series of standard agents (Directory Facilitator, Agent Management System) according to the FIPA standard whose mission is to register and disseminate the services.

\section{ACKNOWLEDGEMENTS}

This work has been supported by the Ministerio de Economía y Competitividad (Spain), project TIN2013-40982-R. Project co-financed with FEDER funds.

\section{REFERENCES}

[1]. Z. Ren and C. . Anumba, "Multi-agent systems in construction-state of the art and prospects," Autom. Constr., vol. 13, no. 3, pp. 421-434, May 2004.

[2]. S. Sharples, V. Callaghan, and G. Clarke, "A multi-agent architecture for intelligent building sensing and control," Sens. Rev., vol. 19, no. 2, pp. 135-140, Jun. 1999.

[3]. P. Davidsson and M. Boman, "A multiagent system for controlling intelligent buildings," in Proceedings Fourth International Conference on MultiAgent Systems, 2000, pp. 377-378.

[4]. S. Wang, Z. Xu, H. Li, J. Hong, and W. Shi, "Investigation on intelligent building standard communication protocols and application of IT technologies," Autom. Constr., vol. 13, no. 5, pp. 607-619, 2004.

[5]. J. K. W. Wong, H. Li, and S. W. Wang, "Intelligent building research: a review," Autom. Constr., vol. 14, no. 1, pp. 143159, 2005.

[6]. Z. Ren, F. Yang, N. M. Bouchlaghem, and C. J. Anumba, "Multi-disciplinary collaborative building design-A comparative study between multi-agent systems and multi-disciplinary optimisation approaches," Autom. Constr., vol. 20, no. 5, pp. 537-549, Aug. 2011.

[7]. E. A. Pärn, D. J. Edwards, and M. C. P. Sing, "The building information modelling trajectory in facilities management: A review," Autom. Constr., vol. 75, pp. 4555, Mar. 2017.

[8]. C. Rizo Maestre and F. J. Mora Lizán, "Intelligent Buildings: Considerations for its Design using Multiagent Systems," Int. J. Eng. Res. Technol., no. Volume. 6-Issue.
04, April-2017, 2017.

[9]. "Foundation for Intelligent Physical Agents," 1996. [Online]. Available: http://www.fipa.org/. [Accessed: 17-Mar2017]. 